\title{
Assessment of the aerodynamic response of bridge decks by means of 2D Reynolds Averaged Navier-Stokes simulations
}

\author{
F. Nieto, S. Hernández, I. Kusano \& J. Á. Jurado \\ School of Civil Engineering, University of La Coruña, Spain
}

\begin{abstract}
The results of several bridge decks 2D steady and unsteady RANS (Reynolds Averaged Navier-Stokes) simulations are reported in this work. The focus has been put on the potential of CFD based simulations in industrial applications in the realm of bridge engineering aerodynamic and aeroelastic problems. The force coefficients of a box deck have been computed at different angles of attack finding a good match with the experimental data available in the literature. Also CFD steady simulations have been used to obtain the force coefficients of a $\Pi$ section considered in the original design proposal of a cable-stayed bridge. Based on the numerical computations, modifications in the original design have been proposed adding fairings and baffles which showed an improved aerodynamic response. The scale effects in the performance of guide vanes for mitigating the vortex-induced response of a twin box girder have been analyzed by means of CFD obtaining a good agreement with experimental measurements. Finally the effect of wind barriers in a twin box deck have been assessed based on the spectrum of the lift coefficient for both the cross-section fitted with or without wind barriers.
\end{abstract}

Keywords: CFD, bridge deck, RANS, URANS, force coefficients, vortex shedding.

\section{Introduction}

The use of CFD (Computational Fluid Dynamics) is steadily progressing in its way towards its general use in bridge design problems. From the late 1990s the number of references dealing with the numerical simulations of aerodynamic and aeroelastic problems in bridges has certainly increased and the inherent difficulty 
in the addressed problems and the accuracy in the simulations has definitely improved. On the other hand, the references reporting cases where CFD results have been applied in industrial applications of bridge engineering are scarce [1-3] since this keeps on being a challenge in spite of the recent advances in this field. There are several reasons for this lack of penetration of numerical simulations in real bridge projects. In first place, CFD modelling requires a level of expertise in fluid mechanics, bridge and wind engineering, and CFD solvers which only recently is being transferred to the industry. Besides this, the demand of computer power resources has been always high and cluster technology has been available and affordable for the industry only recently. Finally, the cost of licenses has prevented the massive use of general purpose commercial solvers for bridge engineering problems outside research or very specific high profile projects. In this respect, the introduction of open source solvers has made easier the access to this technology.

In this work the authors are going to summarize a number of CFD based applications in aerodynamic problems of bridge decks which they have been involved with in recent years. In some cases the motivation for the work has been mainly linked with research and in some others, the work has been developed in the frame of technology transfer agreements with the industry. In any case, the practical implications of the works reported herein will be highlighted in spite of the primary purpose of the work, since the main goal of this work is to show the potential of CFD based techniques in bridge engineering applications.

\section{Assessment of the aerodynamic response of bridge decks}

In industrial applications, at the early design stage, force coefficients of the deck cross-section are the primary source of information in order to judge the feasibility of a particular design. Also the analysis of the vortex shedding response can provide valuable information concerning the vortex-induced excitation risk and the possible mitigation strategies by means of aerodynamic appendages.

\subsection{Force coefficients}

Force coefficients are defined as follows:

$$
C_{d}=\frac{D}{\frac{1}{2} \rho U^{2} B} \quad C_{l}=\frac{L}{\frac{1}{2} \rho U^{2} B} \quad C_{m}=\frac{M}{\frac{1}{2} \rho U^{2} B^{2}}
$$

In the former expressions $D$ is the mean drag force per span length, $L$ is the mean lift force per span length and $M$ is the mean twist moment per unit of span length, positive clockwise. $U$ is the flow velocity, $\rho$ is the air density and $B$ is a reference dimension, the deck width in this case. 
Force coefficients are particularly important for assessing the aerodynamic response of bridge decks [4]. They allow identifying the average load caused by the wind on the deck. Consequently, force coefficients also permit quantifying the effect of certain aerodynamic devices such as wind barriers. Moreover, they are also present in the expression of buffeting forces; therefore studies of bridge behaviour under turbulent flow require this piece of information. Force coefficients also provide qualitative information about the expected flutter performance of a deck cross-section, as to avoid one degree of freedom instability the first derivative of lift and moment coefficients must be positive according to the criteria of figure 1; additionally, the greater the value of those derivatives is the lower the flutter critical wind speed of the structure is.

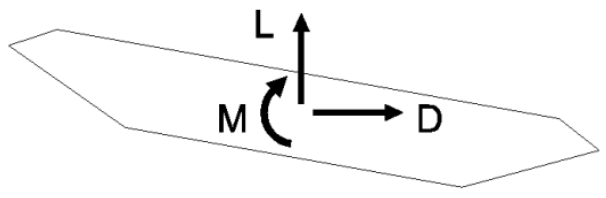

Figure 1: Sign convention for the force coefficients.

\subsection{Vortex shedding}

At the early design of the bridge deck it is very important to take into account the risk of vortex induced excitation at low wind speed. Unsteady simulations considering the static section allow identifying the existence of vortex shedding, its frequency and the effect caused by aerodynamic appendages such as guide vanes or wind barriers. These preliminary assessments must be confirmed latter by wind tunnel tests at the final design evaluation, studying specifically the vortex-induced vibration response of the deck.

\section{Outline of the numerical formulation}

The time averaging of the equations for conservation of mass and momentum gives the Reynolds averaged equations of motion in conservation form [5]:

$$
\begin{gathered}
\frac{\partial U_{i}}{\partial x_{i}}=0 . \\
\rho \frac{\partial U_{i}}{\partial t}+\rho U_{j} \frac{\partial U_{i}}{\partial x_{j}}=-\frac{\partial P}{\partial x_{i}}+\frac{\partial}{\partial x_{j}}\left(2 \mu S_{i j}-\rho \overline{u_{\imath}^{\prime} u_{j}^{\prime}}\right) .
\end{gathered}
$$

where $U_{i}$ is the mean velocity vector, $x_{i}$ is the position vector, $t$ is the time, $\rho$ is the fluid density, $u_{i}^{\prime}$ is the fluctuating velocity and the over-bar represents the time average, $P$ is the mean pressure, $\mu$ is the fluid viscosity, $S_{i j}$ is the mean strain-rate tensor. From the former equation, the specific Reynolds stress tensor is defined as: 


$$
\tau_{i j}=-\overline{u_{\imath}^{\prime} u_{\jmath}^{\prime}}
$$

which is an additional unknown to be modeled based on the Boussinesq assumption for one and two equation turbulence models.

$$
\tau_{i j}=2 v_{T} S_{i j}-\frac{2}{3} k \delta_{i j}
$$

where $v_{T}$ is the kinematic eddy viscosity, $S_{i j}$ is the mean strain-rate tensor and $k$ is the kinetic energy per unit mass of the turbulent fluctuation.

In the examples that are going to be presented next the closure problem has been solved applying k- $\varepsilon$ and both Wilcox`s and Menter's k- $\omega$ SST models for incompressible flows.

\section{Computational approach}

In the sections ahead the results of several simulations conducted using 2D RANS and 2D unsteady RANS are going to be presented. Since the goals of these simulations have been very different and they have been completed in different moments along the latest six years it is not possible to fully describe the characteristics and data of the numerical approach adopted in all of the simulations since it has not always been the same. In the following some general characteristics of the numerical setup are going to be described. Details of each particular simulation can be found in the references provided with the description of the results of each particular case.

The flow domain has been always considered as 2D in order to avoid the high computer power demands of burdensome 3D simulations. The chosen geometry for the domain has been rectangular. In figure 2 a generic flow domain is presented. A velocity inlet is defined at the windward side of the domain and a pressure outlet at atmospheric pressure is set at the leeward end.

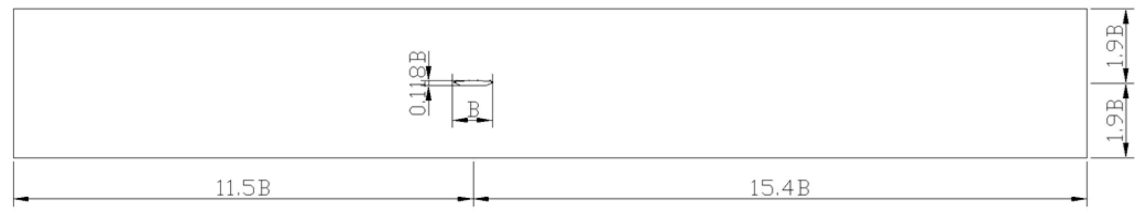

Figure 2: Flow domain geometry.

Low turbulence flow has been generally considered, with typical values of turbulent intensity around $1 \%$ and length scale about $0.1 \mathrm{~B}$. A refined structured mesh is defined around the bride deck with enhanced wall treatment. In general, a non-structured pave type mesh has been defined in the main flow domain. 


\section{Force coefficients of a box deck}

The force coefficients of the box girder of the Normandy Bridge have been obtained by means of 2D RANS simulations adopting k- $\omega$ SST turbulence model $[6,7]$, which has offered better results in bridge engineering problems than $k-\varepsilon$ turbulence model [8].

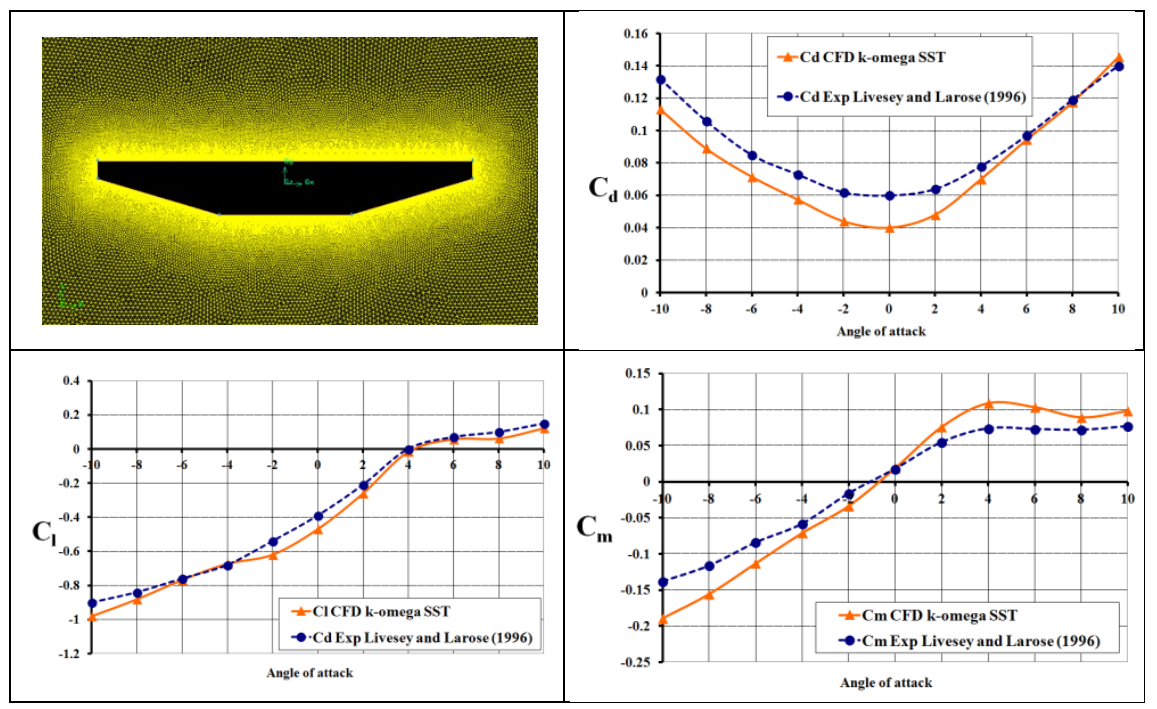

Figure 3: Mesh detail and force coefficients for the Normandy Bridge.

Figure 3 shows the ability of the inexpensive steady state 2D RANS simulations to correctly reproduce the values and the slopes of the force coefficients, even in the range of angles of attack where the values are nearly constant. This type of simulation allows a quick assessment of the mean action on the deck or the expected aeroelastic response of the structure based on the values and signs of the lift and moment force coefficients.

\section{Shaping the cross-section of a cable-stayed bridge girder}

In the following the CFD based modifications proposed in the design of the cross-section of a cable stayed bridge are going to be reported. Further information can be found in [9]. In the frame of the studies for the initial design of a $425 \mathrm{~m}$ length cable-stayed bridge, the aerodynamic response of a $\Pi$ type cross section depicted in figure 4 wished to be assessed. The cross-section is asymmetric since the layout of the bridge was parallel and adjacent to an already existent bridge, located at the right side in figure 4 . In all the studies the flow has been assumed as undisturbed, coming from the left side according to figure 4 .

It is well know that $\Pi$ type cross-sections do not provide a feasible aerodynamic and aeroelastic response, therefore the expected aerodynamic 
performance of the original design should be assessed and modifications in the design proposed if needed. Due to the tight time schedule for providing results it was not possible to conduce wind tunnel tests, thus a CFD based approach was the only available alternative. In order to avoid high computer times a steady 2D RANS approach was chosen for the initial set of simulations.

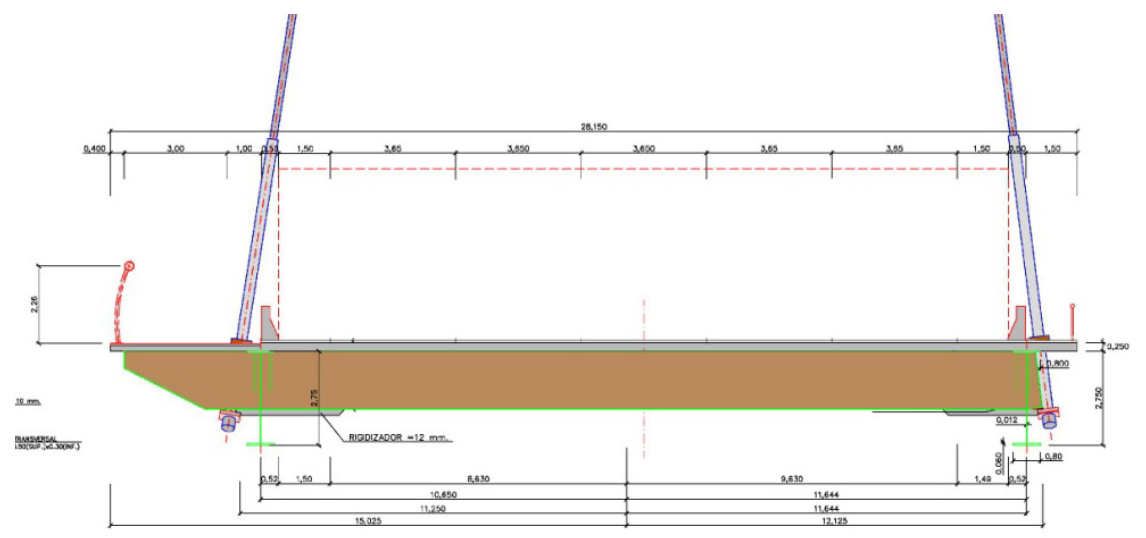

Figure 4: Original design of the cross-section a $425 \mathrm{~m}$ cable-stayed bridge.

One of the key issues in this kind of problems is setting mesh characteristics and a computational approach capable of providing results accurate enough for the purpose of the simulation. Due to the absence of wind tunnel tests of the original design for validating the numerical computations, the cross section was modified in order to obtain a geometric arrangement similar to the one in published references reporting wind tunnel tests data. In figure 5 the force coefficients of the original cross-section with a shorter windward overhang are compared with experimental data in [10] for an overhang to depth ratio $C / D=0.5$. It must be noticed that in reference [10] no barriers are considered on the deck, therefore discrepancies between numerical and experimental data are to be expected. Besides this, the width to depth ratio of the cross-section (without barriers) is about 8.5 while the cross-section in [10] this ratio is 10 .

The results in figure 5 allow the validation of the numerical simulation reported here in. The discrepancies in the force coefficients can be explained due to the effect of the existing barriers in the studied cross-section. However, the steady simulations show their ability to correctly provide mean values for the force coefficients as well as the slopes of lift and moment. Once the adequacy of the mesh and numerical approach has been showed, the force coefficients for the original section have been computed. The results in figure 6 show negative slopes in both lift and moment coefficients which are indicative of one degree of freedom flutter. With the aim of improving the expected aerodynamic response several alternative designs were studied using CFD. The modifications in the design comprised adding fairings and baffles with different separation ratios. In 
figure 6 the force coefficients of the original design and the proposed modified design are presented. It is clear the improvement obtained in terms of slopes of lift and moment coefficients.

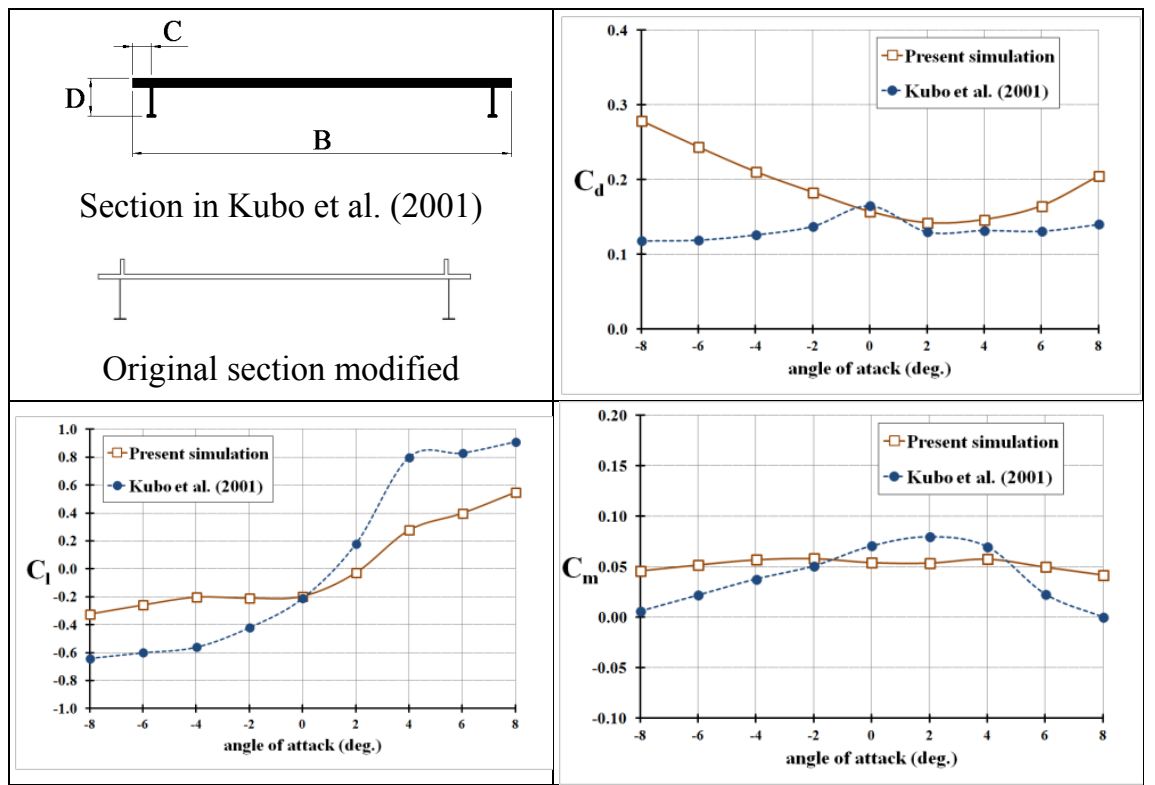

Figure 5: Original design of the cross-section a $425 \mathrm{~m}$ cable-stayed bridge.

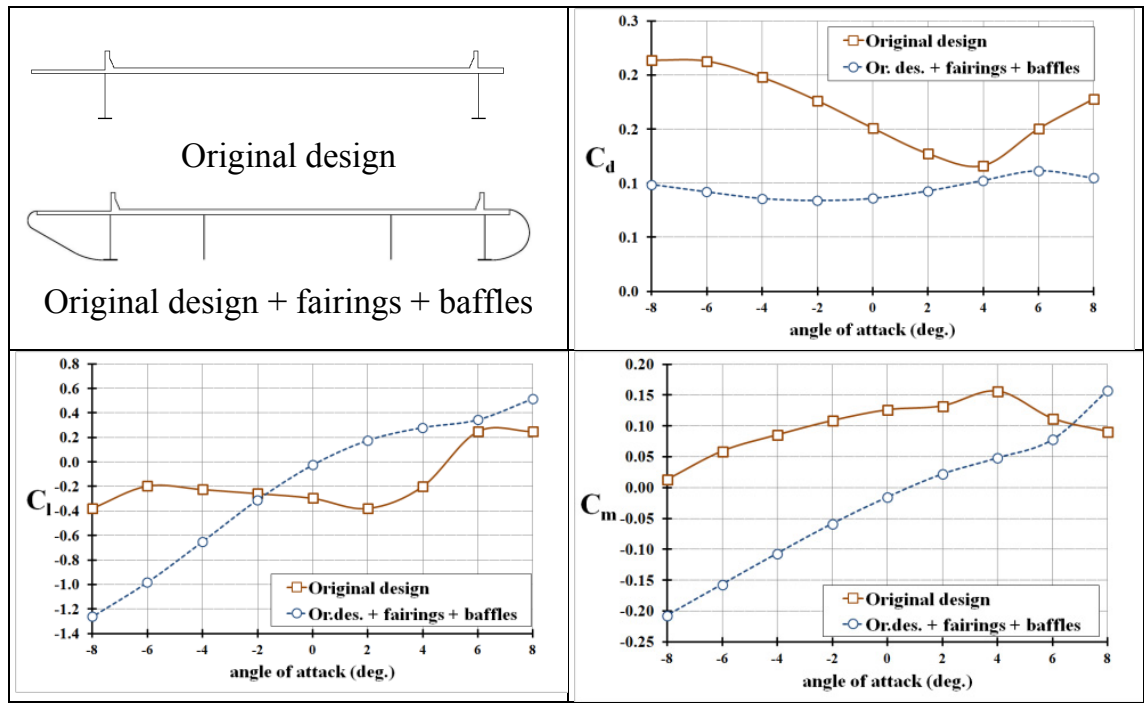

Figure 6: Force coefficients of the original design and the original design fitted with fairings and baffles. 
Vortex shedding response of the cross-section has also been studied by means of unsteady 2D RANS simulations at $0 \mathrm{deg}$. angle of attack. It has been found that strong vortices were shed for the original design, with a Strouhal number of 0.11. This is the same value reported in [11] for the first Tacoma Narrows Bridge cross-section, which is similar to the so-called original design in this work. On the other hand, the proposed design with fairings and baffles did not show any vortex shedding when the unsteady response was computed for a set of different flow velocities.

\section{Performance of guide vanes for mitigating vortex shedding in a twin box deck}

Twin box decks offer a notable performance in terms of flutter instability; however they are prone to vortex induced vibrations. In [12] the experimental tests conducted during the design of the Stonecutters Bridge with the aim of studying the mitigation of the vortex induced vibrations by means of guide vanes have been reported, and it has been remarked the need for conducting the wind tunnel tests at a sufficiently high Reynolds number to avoid thick boundary layers which make inefficient the guide vanes when the tests are performed at low Reynolds numbers. As a further form of cooperation between CFD and wind tunnel testing several 2D URANS simulations have been conducted at different Reynolds numbers for a static twin box deck in order to ascertain the potential of numerical techniques to identify beforehand the required Reynolds number to avoid scale effects when checking the performance of guide vanes. More details can be found in [13].

In figure 7 measurements of the boundary layer upstream of the upwind guide vane are reported for different Reynolds numbers, finding results close to the experimental ones reported in the literature. Therefore, it can be identified by means of CFD simulations the Reynolds number in the wind tunnel tests that satisfies the condition that the displacement thickness must be about $10 \%$ of the guide vane offset.

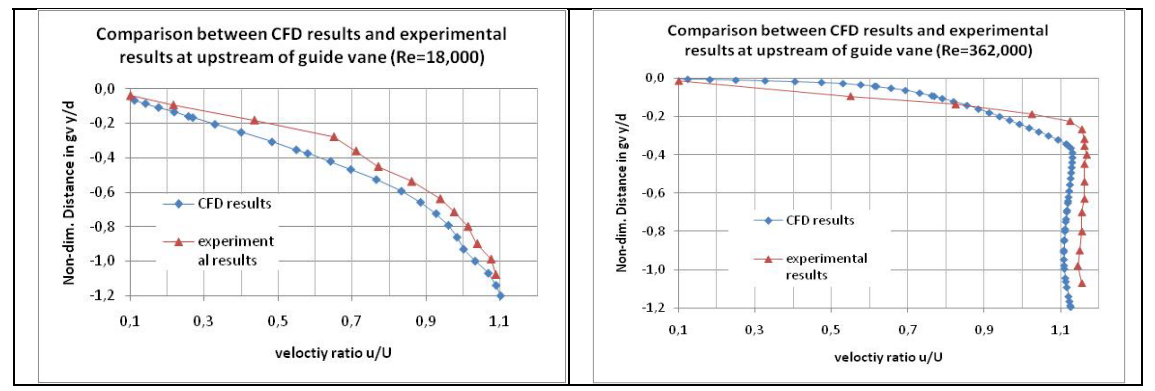

Figure 7: Boundary layers at different Reynolds numbers. 


\section{Performance of wind barriers for mitigating vortex shedding in a twin box deck}

It has just mentioned that twin box decks are vortex-induced vibration prone cross-sections. Besides this, wind barriers are increasingly present in long span bridges due to its shelter effect for traffic, which allows a more comfortable driving and a decrement in the number of bridge closure hours due to high winds. Another important issue to deal with when analyzing the effect of wind barriers is the increment in drag forces due to wind. These forces must be considered since they are finally transmitted to the towers and their foundations. However, another positive effect of wind barriers is their ability to mitigate vortex shedding, as it has been reported, for instance by Ge et al. [14] for the Xihoumen Bridge. In figure 8 the spectrums of the lift coefficient for the twin box arrangement considered at the early design stage of the Forth Replacement Crossing with and without wind barriers are presented. It can be seen how the strong peak at a Strouhal number of 0.16 for the static deck has

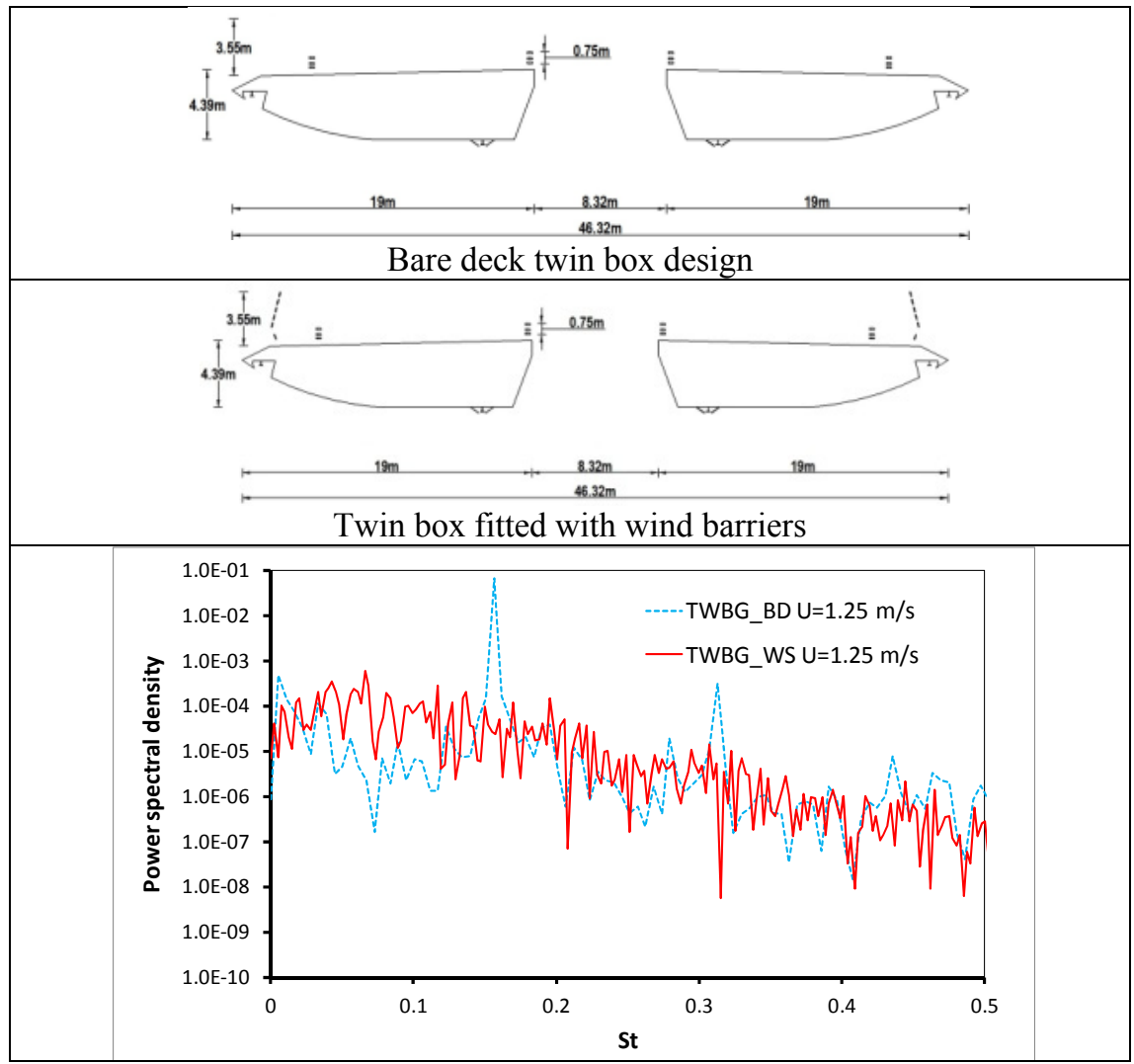

Figure 8: Effect of wind barriers in the lift coefficient spectrum. 
vanished for the case with wind barriers. This remarks the capability of $2 \mathrm{D}$ URANS simulations for assessing the aerodynamic performance of bridge deck designs [15].

\section{Conclusions}

Several 2D steady and unsteady RANS simulations of bridge decks have been presented. The focus has been put on the applicability of CFD based simulations in industrial applications in bridge engineering. It has been shown the ability of steady simulations to accurately provide the mean force coefficients for different cross-sections at different angles of attack. It has also been highlighted the importance of force coefficients to preliminary assess the aeroelastic response of bridge deck and how the design can be improved based on this information.

Unsteady simulations have provided the response in terms of vortex shedding for the static decks. The CFD simulations have been used to preliminary ascertain the effect of fairings and baffles, guide vanes or wind barriers in the mitigation of this effect. It must also be noted how CFD simulations can shed light on scale effects in wind tunnel tests.

CFD based techniques have a great future in the simulation of aerodynamic and aeroelastic effects in bridge engineering. The improvements in modeling formulation, computer power and software are in the way to change the standard procedures in the wind design of long span bridges.

\section{References}

[1] Vejrum, T., Queen, D.J., Larose, G.L., Larsen, A. (2000) Further aerodynamic studies of Lions' Gate Bridge -3 lane renovation. Journal of Wind Engineering and Industrial Aerodynamics; 88, 325-341.

[2] Taylor, I.J., Vezza, M., Salisbury, I. (2008) Numerical investigation of the effects of pedestrian barriers on aeroelastic stability of a proposed footbridge. Journal of Wind Engineering and Industrial Aerodynamics; 96, 2418-2437.

[3] Sarwar, M.W., Ishihara, T. (2010) Numerical study on suppression of vortex-induced vibrations of box girder bridge section by aerodynamic countermeasures. Journal of Wind Engineering and Industrial Aerodynamics; 98, 701-711.

[4] Diana, G., Fiammenghi, G. (2012) Wind tunnel tests and numerical approach for long span bridges: the Messina bride. In Proc. of the Seventh International Colloquium on Bluff Body Aerodynamics and its Applications (BBAA7). Shanghai, China.

[5] Wilcox, D.C. (2006) Turbulence modeling for CFD. 3rd Edition; DCW Industries, Inc.

[6] Nieto, F., Jurado, J.Á., Hernández, S. (2008) Obtención de coeficientes aerodinámicos mediante CFD en secciones transversals de tableros de puentes de hormigón. In Pro. Of the IV Congreso de ACHE. Valencia, España. (In Spanish). 
[7] Livesey, F.M., Larose, G.L. (1996) The Pont de Normandie during construction, aeroelastic modelling of behaviour. Journal of Wind Engineering and Industrial Aerodynamics, 68; pp.: 203-2015.

[8] Sun, D., Wright, N.G., Owen, J.S., Liaw, K. (2005) Identification of 18 flutter derivatives using CFD turbulence modelling. Proc. of the $4^{\text {th }}$ European \& African Conference on Wind Engineering. Prague, Czech Republic.

[9] Nieto, F., Hernández, S., Jurado, J.Á., Baldomir, A. (2010) CFD practical application in conceptual design of a $425 \mathrm{~m}$ cable-stayed bridge. Wind and Structures, 13(4) pp.: 309-326.

[10] Kubo, Y., Sadashima, K., Yamaguchi, E., Kato, K., Okamoto, Y., Koga, T. (2001) Improvement of aeroelastic instability of shallow $\Pi$ section. Journal of Wind Engineering and Industrial Aerodynamics, 89, pp.: 1445-1457.

[11] Larsen, A., Walther, J.H. (1998) Discrete vortex simulation of flow around five generic bridge deck sections. Journal of Wind Engineering and Industrial Aerodynamics; 77-78, 591-602.

[12] Larsen, A., Savage, M., Lafrenière, A., Hui, M.C.H., Larsen, S.V. (2008) Investigation of vortex response of a twin box bridge section at high and low Reynolds numbers. Journal of Wind Engineering and Industrial Aerodynamics, 96, pp.: 934-944.

[13] Nieto, F., Kusano, I., Hernández, S., Jurado, J.Á. (2010) CFD analysis of the vortex-shedding response of a twin-box deck cable-stayed bridge. In Proc. the Fifth International Symposium on Computational Wind Engineering. North Carolina, USA.

[14] Ge, Y., Yang, Y., Cao, F. (2011) VIV sectional model testing and field measurement of Xihoumen Suspension Bridge with twin box girder. In Proc. of the $13^{\text {th }}$ International Conference on Wind Engineering. Amsterdam, The Netherlands.

[15] Nieto, F., Hernández, S., Kusano, I., Jurado, J.Á. (2012) CFD aerodynamic assessment of deck alternatives for a cable-stayed bridge. In Proc. of the Seventh International Colloquium on Bluff Body Aerodynamics and its Applications (BBAA7). Shanghai, China. 